\title{
Hypertrichosis cubiti (hairy elbows) and short stature: a recognisable association
}

\author{
K D MACDERMOT*, M A PATTON $\dagger$, M J H WILliaMS $\ddagger$, AND R M WINTER* \\ From * the Kennedy Galton Centre, Northwick Park Hospital, Watford Road, Harrow, Middlesex HA1 3UJ; $\vec{\circ}$

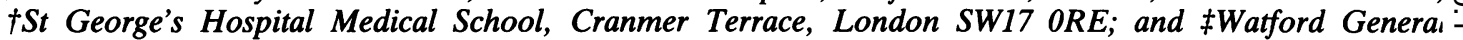 \\ Hospital, Vicarage Road, Watford, Herts.
}

SUMMARY We report four patients with hypertrichosis cubiti who were referred for investigation $\dot{\sim}$ of short stature. Two males, whose height was on and just below the 3rd centile respectively, $\dot{\sigma}$ were sporadic cases and two females with disproportionate short stature were mother and $\dot{\omega}_{\infty}$ daughter. Radiological changes present in the familial cases were non-specific and biochemical $N$

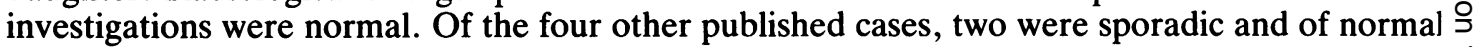
height. The other two were sibs with short stature and their parents were heterozygous for the $\vec{c}$ Weill-Marchesani syndrome. We were unable to ascertain whether hypertrichosis cubit cosegregates with the same type of skeletal dysplasia or elucidate the type of genetic transmission of hypertrichosis cubiti alone.

Hypertrichosis cubiti was first reported by Beighton, ${ }^{1}$ who described a localised hypertrichosis confined to the extensor surface of the elbow regions in two sibs from an inbred Amish family. They had normal development and clinical abnormalities were confined to short stature and foreshortened, but not dysplastic, nails. Their parents were short and both were heterozygous for the Weill-Marchesani syndrome. The short stature of the affected sibs was not investigated. Subsequently, Rudolph ${ }^{2}$ and Andrev and Stansky ${ }^{3}$ each reported a sporadic case of hypertrichosis cubiti without other clinical abnormalities. Sighting of a case in the local parish church congregation was reported by Warner ${ }^{4}$ and a few cases were apparently observed, but not published. $^{3}$

We have had the opportunity to examine four previously unreported patients with hypertrichosis cubiti, all of whom were referred for investigation of short stature.

\section{Case reports}

Two males and two females presented with a history of excessive hair growth over the lateral aspects of the lower third of the upper arm and upper third of the forearm observed soon after birth. In early childhood the hair became coarser, sometimes

Received for publication 29 October 1988.

Revised version accepted for publication 12 January 1989.

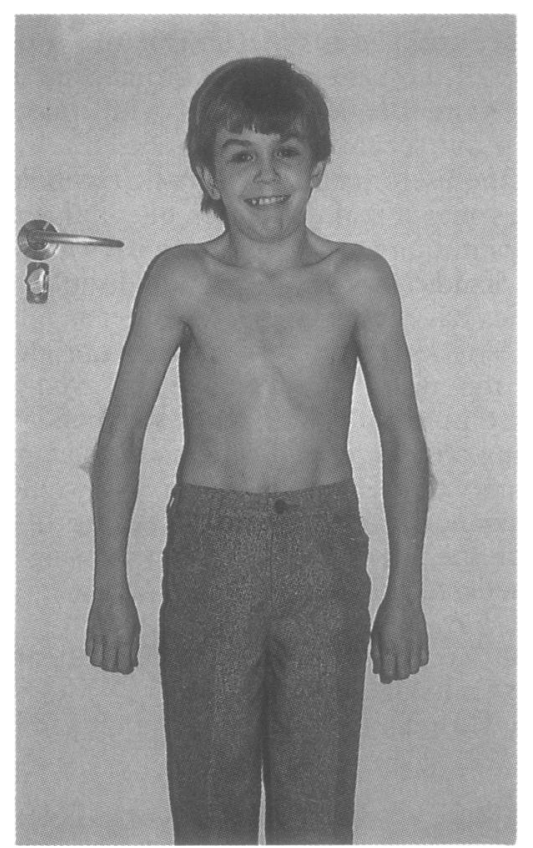

FIG 1 Case 1. Sporadic case of hypertrichosis cubiti with the height just below the 3rd centile. Positional elevation of the shoulders. 
curly, pigmented, and lengthened to 5 to $8 \mathrm{~cm}$. The underlying skin was normal. They had a normal amount of body hair and no other areas of hypertrichosis. The only adult patient seen reported abatement of this localised excessive hair growth around puberty and, when examined, it was no longer apparent. All patients had normal birth weight, psychomotor development, and clinical systems examination. They were in good health and took no medication.

Case 1 (fig 1) was a $12^{1 / 2}$ year old male. His height was $132 \mathrm{~cm}$ (<3rd centile), with growth velocity just below the 3rd centile for the last three years. Mid parental height could not be estimated as he was adopted. However, his foster parents, who also adopted his brother and sister, reported that his parents and sibs have normal stature. The skeletal survey was normal. There was no family history of hypertrichosis.

Case 2 (figs 2 and 3) was a seven year old male with a height of $109 \mathrm{~cm}$ (3rd centile), compatible with the predicted height from mid parental value. The height of his three and a half year old brother was also on the 3 rd centile. The skeletal survey was

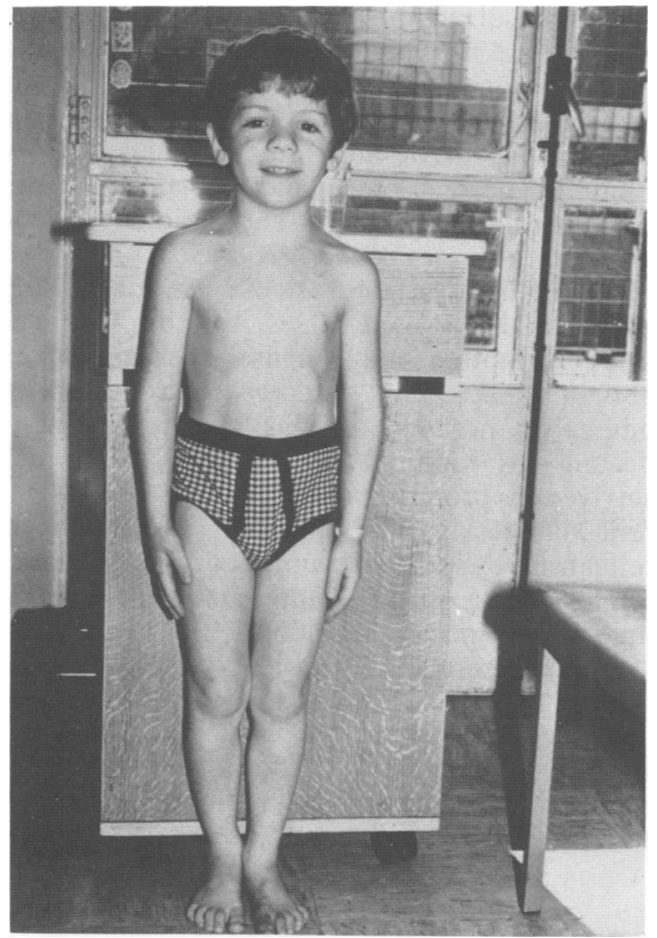

FIG 2 Case 2. Antimongoloid slant of the eyes, mild hypertelorism, and foreshortened nose.

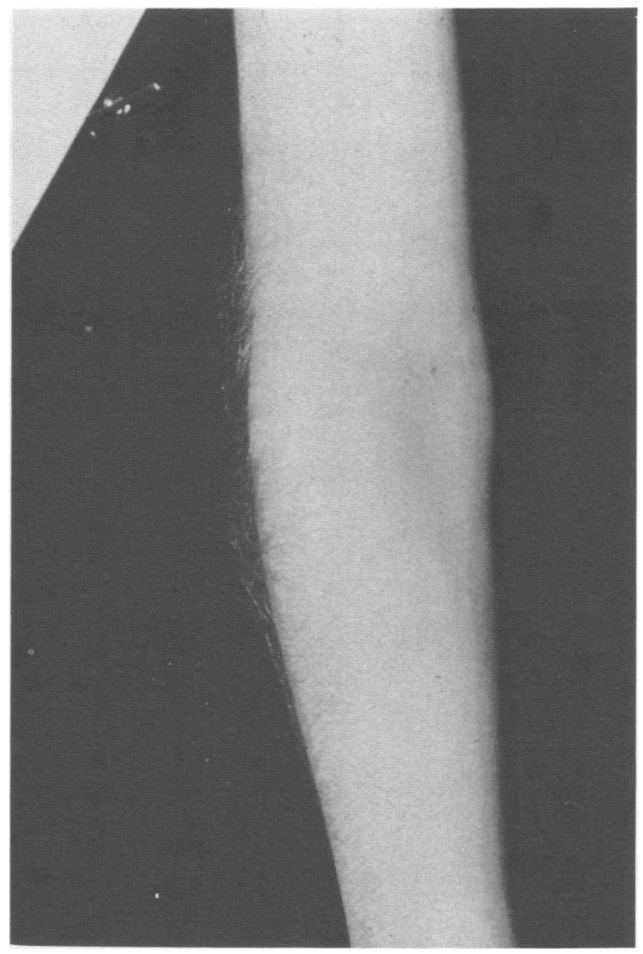

FIG 3 Case 3. Hypertrichosis cubiti.

normal. He had an antimongoloid slant of his eyes, mild hypertelorism, and a short nose with thick alae. There was no family history of hypertrichosis.

Cases 3 and 4 (fig 4) are mother and daughter, respectively, with marked shortness of stature. Neither of them had any sibs. In the pedigree, there were no other persons with short stature or hypertrichosis.

Case 3 was noted to be short at 18 months but the measurement obtained is not available. At the age of eight years three months her height was $101 \mathrm{~cm}$ ( $>3$ SD below the mean). On examination she appeared to have rhizomelic shortening of the extremities. Skeletal survey showed a number of minor abnormalities. She had delayed bone age which was five years nine months at the age of eight years three months, some shortening of her metacarpals, and clinodactyly. Metaphyses were flared and bone modelling was normal. There was acetabular dysplasia and the symphysis pubis was a little wide with short ischia. The capital femoral epiphyses were small and the vertebral bodies slightly oval. The distal radial epiphyses sloped. The skull vault appeared relatively large. She had a round face 


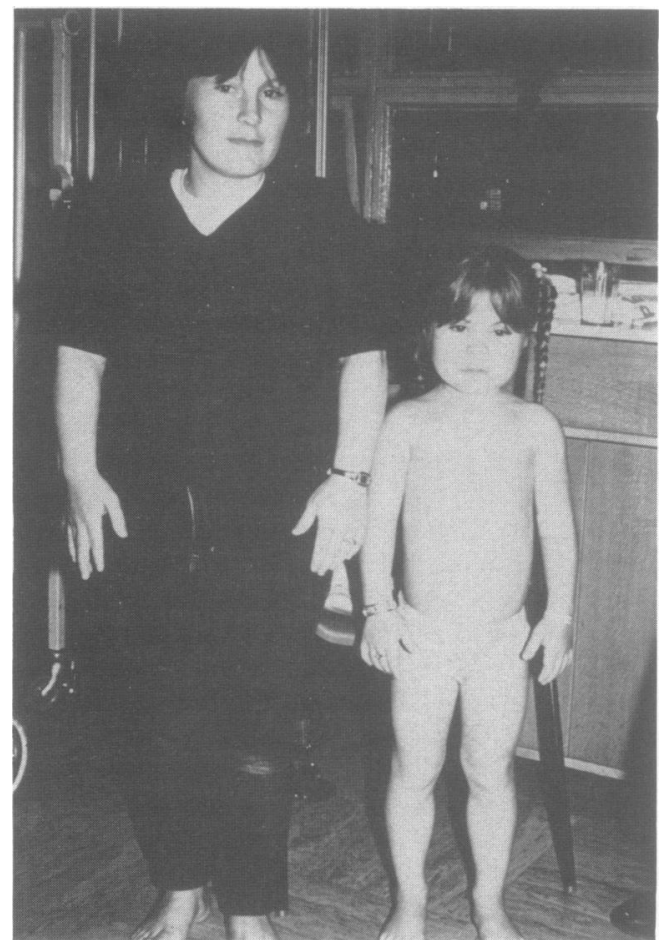

FIG 4 Cases 3 and 4. Mother and daughter with hypertrichosis cubiti, short stature, rhizomelic shortening of the extremities, round facies, and a heavy jaw.

with epicanthic folds, mild hypertelorism, a low nasal root, and a foreshortened nose.

Case 4 was noted to be short in early childhood and her adult height was $130 \mathrm{~cm}$ ( $>3$ SD below the mean). Rhizomelic limb shortening was apparent. Skeletal survey showed normal bone modelling and minor abnormalities confined to the spine. She had narrow interpedicular distances in the lumbar region and short pedicles. Mild scoliosis with some vertebral end palate irregularity was present in the dorsal region. She had a round face with prominent jaw.

The following investigations were normal in all cases: chromosome karyotype, routine haematology and biochemical profile, total T4, THBI, free T4 index, and TSH. All patients except case 1 (not measured) had normal TRH and hypoglycaemia stimulated growth hormone assay.

\section{Discussion}

Generalised hypertrichosis is most commonly seen in certain racial groups and falls within the normal range of hair distribution. Pathological generalised $\stackrel{\vec{\rho}}{\vec{g}}$ hypertrichosis with predilection for certain sites is associated with hyperactivity of the adrenal cortex $\frac{C}{0}$ or tumours producing excess steroids or androgens. $\bar{c}$ Drugs (valproic acid) and metabolic disorders (porphyria, mucopolysaccharidosis) produce hypertrich- 을 osis by an as yet unknown mechanism. Generalised $\omega$ congenital hypertrichosis lanuginosa results from a $\overrightarrow{0}$ rare autosomal dominant gene. Localised hypertrichosis is commonly seen over a naevus or spina bifida.

The only biopsy report of the hairy site in hypertrichosis cubiti ${ }^{3}$ showed no structural abnor- i malities of the hair follicles. The faster than normal $\sigma$ hair growth was reflected by the finding of $90 \%$ of $\omega$ the hair follicles being in the anagen phase, $9 \%$ in $N$ the telogen, and $1 \%$ in the catagen phase. ${ }^{3}$ Andreev 웅 and Stransky ${ }^{3}$ concluded that it probably repre- $\rightarrow$ sented a naevoid condition of the hair follicles. $\overleftarrow{c}$ Urinary 17-ketosteroids and 17-hydrocortisone, $\overparen{\mathbb{D}}$ plasma cortisol, and testosterone were measured in one case $^{3}$ and were normal. All patients so far reported, including our cases, simply improved their cosmetic appearance by regular cutting of the excess hair.

The unresolved questions regarding hypertrichosis cubiti are its type of genetic transmission and a possible association with short stature. The four familial cases have severe shortening of stature, while the height of the three sporadic cases was within the normal range and just below the 3rd centile in the fourth case. The aetiology of short stature in our cases 3 and 4 is unclear. Hypochondroplasia and dyschondrosteosis were considered and excluded. Round facies, obesity, and short stature in mother and daughter are suggestive of Albright's hereditary osteodystrophy but $x$ ray findings do not support this diagnosis. The two affected sibs reported by Beighton ${ }^{1}$ had brachydactyly and proportionate short stature, seen in Weill-Marchesani syndrome, but the results of a skeletal survey were not published. It is uncertain if skeletal changes are comparable in these two ? families or coincidental findings.

Genetic heterogeneity in the type of transmission of hypertrichosis cubiti may exist. Autosomal dominant inheritance is most probable in cases 3 and 4 (mother and daughter). In the two sibs reported by Beighton ${ }^{1}$ from the Amish community, autosomal recessive inheritance is more likely.

We are grateful to Dr S Greene for referring cases 3 and 4 and to Mrs J Bourne for typing this manuscript. 


\section{References}

1 Beighton P. Familial hypertrichosis cubiti: hairy elbows syndrome. J Med Genet 1970;7:158-60.

2 Rudolph RI. Hairy elbows. Cutis 1985;36:69.

3 Andreev VC, Stransky L. Hairy elbows. Arch Dermatol 1979:115:761.
${ }^{4}$ Warner TFCS. Hairy elbows. Arch Dermatol 1980;116:19.

Correspondence to Dr K D MacDermot, Kennedy Galton Centre, Northwick Park Hospital, Watford Road, Harrow, Middlesex HA1 3UJ. 